\title{
O Pensamento Computacional como base para o ensino-aprendizagem de matemática através da OBMEP
}

\author{
Natália Bernardo Nunes², Lucas Pinheiro Alves ${ }^{1}$, Aline Silva De Bona ${ }^{1}$ \\ ${ }^{1}$ Instituto Federal de Educação, Ciência e Tecnologia do Rio Grande do Sul - Campus \\ Osório. R. Santos Dumont, 2127 - Albatroz, Osório - RS \\ ${ }^{2}$ Instituto Federal de Educação, Ciência e Tecnologia Sul-rio-grandense - Campus Passo \\ Fundo. Estrada Perimetral Leste, 150 - São José, Passo Fundo - RS \\ nataliabernunes@gmail.com, lucaspalves8@gmail.com, \\ aline.bona@osorio.ifrs.edu.br
}

\begin{abstract}
In front of the less interested students for mathematics and the educational insights below expected, this article reports a part of an action-research that uses the computational thinking pillars behind autoral questions similar to OBMEP, that is in $99 \%$ of the brazilian cities. The search for resources took place through a systematic literature review with referenced articles in the area, followed by the elaboration of the activities. It was elaborated 19 questions with computational thinking, tested with winners students in the olympiad, whereas the analysis of possible cases to direct the clearest resolution.
\end{abstract}

Resumo. Diante do desinteresse pela disciplina de matemática pelos estudantes que atuam e os baixos índices educacionais brasileiros, o presente artigo relata um recorte de uma pesquisa-ação que utiliza os pilares do pensamento computacional através de questões autorais semelhantes às da OBMEP, por estar presente em 99\% dos municípios brasileiros. A busca por recursos se deu por meio de uma revisão sistemática de literatura com artigos de eventos de referência na área, seguida da elaboração das atividades. Foram elaborados 19 problemas envolvendo o pensamento computacional, testadas com estudantes premiados na olimpíada, onde foi observada a análise de casos possíveis para direcionar à resolução mais clara possível.

\section{Introdução}

Fazer com que o estudante se interesse pela aprendizagem da matemática vem se tornando um desafio cada vez maior para professores de todo o Brasil. Como mostrado em um levantamento divulgado pelo Sistema de Avaliação da Educação Básica (SAEB) em 2017, somente 4,52\% dos estudantes do ensino médio superaram o nível 7 da escala de proficiência da avaliação. E a situação piora quando avaliado o $9^{\circ}$ ano do ensino fundamental, onde a maioria dos estudantes atingiu somente o nível 3 da escala de proficiência. A escala de proficiência é dividida entre os níveis 0 a 10.

O desinteresse pelo aprendizado de matemática pode ser explicado no momento em que refletimos sobre métodos utilizados em sala de aula, já que esses não passam por uma grande mudança desde o século XIX, contrastando com a atual realidade do estudante, que pode ser considerado um nativo digital. Segundo [Palfrey e Gasser, 2011] Nativos digitais são pessoas que tiveram contato com recursos digitais desde muito cedo e tem sua vida física e sua vida digital fortemente atreladas, não vendo motivo para separá-las. Visando ajudar a solucionar a problemática aqui apresentada, o presente artigo traz o recorte de uma pesquisa-ação que utiliza o pensamento computacional e 
seus pilares na elaboração de questões autorais inspiradas na OBMEP, tendo em vista que ela está presente em 99\% dos municípios brasileiros e tem entre seus objetivos contribuir para a educação de qualidade e incentivar o aperfeiçoamento de professores.

\section{O pensamento computacional na elaboração de atividades inspiradas na OBMEP}

Uma definição para o termo "Pensamento computacional" é apresentada por (Baar e Stephenson, 2011), onde eles o abordam voltado para o uso em sala de aula, falando do termo como uma metodologia para solucionar problemas de assuntos diversos, possibilitando assim o uso do conceito em diferentes disciplinas.

Conforme sua publicação em 2019, Silva define o pensamento computacional como "o processo de raciocínio baseado em fundamentos computacionais para a resolução de problemas de diversas áreas" [Silva, 2019].

Bobsin e outros (2020) apontam, utilizando como estado da arte dois problemas investigativos, a importância deste tipo de problema nas aulas de matemática, tendo em vista a abordagem do pensamento computacional presente em qualquer conteúdo de qualquer faixa etária do ensino básico quando isso ocorre. $\mathrm{Na}$ mesma linha de raciocínio, as olimpíadas do conhecimento inserem-se como elemento de apoio ao ensino de matemática, devido à grande variedade de problemas investigativos, sendo um assunto de interesse de professores, inclusive para aplicar o pensamento computacional no ensino remoto e híbrido [Nunes, 2021]

Para Vicari e outros (2018), o pensamento computacional é uma metodologia que deve estar presente nas salas de aula apoiado por quatro pilares, sendo eles: Decomposição: Dividir um problema em partes menores; Reconhecimento de padrões: Analisar e identificar pontos em comum de problemas para que possa ser criada uma solução aplicável a todos eles; Abstração: Retirar de um problemas somente os pontos realmente relevantes, excluindo o resto; Algoritmos: Sequência de passos lógicos para resolver um problema. Ademais, os estágios abordados por Guarda e Pinto (2020) também são considerados para o pensamento computacional enquanto metodologia nas aulas de matemática. São eles: Definir o problema; Solução do problema e Analisando a Solução.

Vale também ressaltar que o pensamento computacional e seus pilares são contemplados pela Base Nacional Comum Curricular (BNCC) como parte da disciplina de matemática [BNCC, 2018].

Simultaneamente ao estudo aqui apresentado, ocorre desde o ano de 2005 a aplicação da OBMEP, estando presente em 99\% dos municípios brasileiros, e por este motivo, é de conhecimento comum de estudantes e professores brasileiros. E tem por objetivos "Incentivar o aperfeiçoamento dos professores das escolas públicas, contribuindo para a sua valorização profissional" e "Contribuir para a melhoria da qualidade da educação básica, possibilitando que um maior número de alunos brasileiros possa ter acesso a material didático de qualidade" [OBMEP. 2021]. Segundo dados levantados pelo o Centro de Gestão de Estudo Estratégico em 2011, 38\% dos estudantes afirmam que as questões de OBMEP fizeram-os raciocinar mais. Todavia, segundo a mesma pesquisa, $41 \%$ dos professores afirmaram não ter percebido alteração 
em relação aos estudos de seus alunos devido à olimpíada [CGEE, 2011] e uma das razões para isso pode ser o alto nível de complexidade das questões em relação à abordagem realizada na disciplina de matemática, durante todo o ano letivo nas instituições de ensino.

No entanto, o pensamento computacional vem ganhando força como uma possibilidade de cativar o interesse daqueles que receberão esta abordagem. Pois o pensamento computacional segundo segundo Bobsin, Bona e Kologeski (2020), explora diferentes materiais, recursos e contextos já presentes nas instituições de ensino e/ou conhecimento comum do estudante.

As questões apresentadas pela OBMEP podem ser relacionadas a uma metodologia interessante e bem aceita por professores e estudantes quanto a resolução de problemas, que é a metodologia investigativa de Ponte, Brocardo, Oliveira (2006) e Bona (2016), pois investigar é pesquisar uma curiosidade, é procurar uma resposta para uma pergunta interessante. Pois assim como é trabalhado na metodologia as questões da OBMEP geralmente causam este sentimento em quem está resolvendo. Partindo disto é válido propor uma aplicação em sala de aula de problemas inspirados nos da OBMEP que contemplem o pensamento computacional.

\section{Objetivos}

Autores defendem a presença das olimpíadas para um melhor conhecimento do pensamento computacional e demais conceitos da computação. Contudo, sua utilização ainda é pouco explorada pela comunidade acadêmica. Em uma busca nos anais do Simpósio Brasileiro de Informática na Educação (SBIE) ${ }^{1}$, em todas as suas edições, pelo termo "olimpíada" em títulos, resumos e palavras-chave de suas publicações, apenas 3 trabalhos foram encontrados, sendo todos eles compreendendo a utilização da Olimpíada Brasileira de Informática (OBI) como ferramenta de aprendizagem. Vale ressaltar que houve um intervalo de oito anos entre a primeira e a segunda publicação encontrada, apontando uma lacuna ainda maior na esfera olímpica atrelada ao ensino-aprendizagem da educação básica.

Duarte, Moreira e Mello (2010) defendem que a competitividade pode estimular o aprimoramento e estudo de estudantes e profissionais, contribuindo para formar, inclusive, o que é chamado de alunos atletas. Souza e Lopes (2018) observam características da OBI para trabalhar o pensamento computacional através da computação desplugada utilizando 5 dinâmicas. As autoras relatam que o raciocínio trabalhado nessas dinâmicas estimularam os estudantes a compreender os conceitos da olimpíada. Por fim, Santana, Lopes e Braga (2019) concluíram, por meio de uma análise do uso da OBI em diferentes disciplinas do ensino fundamental, que o desempenho dos estudantes na competição possui uma correlação maior com a disciplina de língua portuguesa, com o domínio de leitura e interpretação de texto.

Diante da situação apresentada, o objetivo principal do presente estudo é como auxiliar professores da disciplina de matemática, estimulada de maneira menos predominante na OBI, a tornarem seus métodos educacionais adequados ao ambiente e

\footnotetext{
${ }^{1}$ Disponível em: <https://br-ie.org/pub/index.php/sbie/index>
} 
à necessidade dos estudantes da atualidade através da tecnologia e utilizando a própria olimpíada de matemática, que também possui um material consistente para trabalhar o pensamento computacional, com atividades lúdicas que abordem o mesmo raciocínio utilizado nas questões da OBMEP. Entretanto, ressalta-se que não é a finalidade deste artigo afirmar que o corpo docente necessita passar por uma atualização, e sim a elaboração de um material didático com as atividades mencionadas aqui. Além de promover uma reflexão quanto a utilização dos materiais fornecidos gratuitamente pela olimpíada para a criação de suas próprias atividades.

Nesse sentido, objetiva-se, no presente artigo, apresentar uma pesquisa de campo com um grupo qualificado de estudantes, ou seja, premiados na OBMEP, diante da resolução de problemas investigativos através de questões semelhantes às da olimpíada que trabalhem os quatro pilares do pensamento computacional. Conforme observado na busca por pesquisas relacionadas, esta análise é inédita, pois avalia a diversidade de resoluções possíveis para um mesmo conteúdo, assim como um mesmo algoritmo pode ser escrito de diferentes maneiras de acordo com o programador que o desenvolve. Assim, visa-se a elaboração de um material didático que servirá de apoio para professores da educação básica, com as atividades aqui mencionadas, entre outras, todas testadas e validadas por alunos e professores da educação básica. Além das atividades, também serão sugeridos materiais para a sua execução.

\section{Metodologia}

A metodologia aplicada para a realização da pesquisa que originou o presente artigo se baseia na pesquisa-ação, onde atividades inspiradas na OBMEP que envolvem o pensamento computacional foram elaboradas e testadas.

Para a elaboração das atividades, foram utilizados como referência as provas da olimpíada em todas as suas edições (de 2005 a 2019) e o livro "Círculos Matemáticos A Experiência Russa” (Fomin et al., 2012), ora utilizando o conteúdo abordado e adaptando a sua metodologia, ora entendendo o raciocínio utilizado na questão e alterando o seu aspecto visual e o conteúdo trabalhado. O pensamento computacional foi inserido pensando nos seus quatro pilares (decomposição, abstração, algoritmos e reconhecimento de padrões), procurando utilizar, sempre que possível, todos eles, de acordo com o que já estava pré-estabelecido na questão. Desta forma, foi possível inserir elementos físicos como tabuleiros confeccionados e material dourado (desplugadas) ou recursos digitais e plataformas online como code.org ${ }^{2}$ e khan academy $^{3}$

A testagem se deu em diferentes etapas, passando por estudantes da escola básica, estudantes do ensino técnico integrado ao ensino médio e professores sendo a descrita no presente artigo a mais recente, abordando como estudantes premiados na olimpíada resolvem as questões criadas anteriormente com base no raciocínio utilizado na OBMEP atrelado ao pensamento computacional. Este grupo qualificado foi escolhido pois são estudantes que possuem experiência na resolução de problemas investigativos através de questões da olimpíada.

\footnotetext{
${ }^{2}$ Disponível em: <http://code.org >

${ }^{3}$ Disponível em: <https://pt.khanacademy.org/>
} 
Para isso, as atividades elaboradas foram adicionadas em um formulário online e distribuída a participantes do Programa de Iniciação Científica Jr, e do Programa Mentores da OBMEP, em parceria com a coordenação acadêmica da OBMEP de estado omitido, através de grupos no WhatsApp. Os estudantes eram convidados a responderem ao formulário com todas as perguntas. Contudo, como o público-alvo abrange uma faixa etária muito diversificada ( $7^{\circ}$ ano do Ensino Fundamental até universitários), nenhuma pergunta era obrigatória, propondo, assim, que eles resolvessem apenas as atividades que se sentissem capazes de formular um raciocínio.

\section{Resultados e discussão}

Ao todo, 19 atividades foram criadas, onde 15 utilizam recursos da computação desplugada e apenas 4 abordando a computação plugada, devido a dificuldade em encontrar recursos gratuitos, acessíveis e abertos para a criação de atividades nas metodologias propostas.

As atividades foram enviadas em 13 turmas do Programa de Iniciação Científica Jr, sendo uma do programa Mentores da OBMEP. A principal característica das resoluções, em comparação com as testagens anteriores realizadas, foi a maneira com que estudantes premiados na olimpíada fazem para chegar até a solução, apresentando, muito além da resposta final, o raciocínio utilizado para a resolução do problema. A atividade a seguir exemplifica a linha de pensamento que um estudante possui em determinada atividade e os pontos identificados do pensamento computacional em sua resolução

\subsection{Exemplo de atividade respondida}

Nesta seção, um exemplo de atividade desenvolvida com motivação em questões da OBMEP é apresentado. A abordagem desplugada apresenta como ele pode ser utilizado nas instituições de ensino, podendo ainda servir como base para uma nova atividade, ou uma continuação da mesma, utilizando, posteriormente, uma resolução de problemas de maneira plugada.

\subsubsection{Enunciado}

Atividade com motivação na questão 14 do nível 3 da Primeira Fase da OBMEP 2018 .

Among Us é um jogo de videogame onde seus jogadores são tripulantes de uma nave e, entre eles existe de um a três impostores que realiza sabotagens e assassina os demais tripulantes. Quando um jogador encontra um corpo na nave, ele pode reportá-lo e todos são direcionados para uma sala de discussão para se defenderem e definirem quem é o impostor. Assim, o jogador mais votado pela tripulação é eliminado da nave.

Lalá, Lelé, Lili, Loló, Lulu e Lilão estão jogando Among Us até que Lilão reporta o corpo de Lelé e os jogadores defendem os seguintes argumentos na discussão:

Lalá: $\mathrm{Eu}$ estava junto com Lulu, e por isso nem eu, nem ela somos os impostores.

Lili: Passei por Lelé um pouco antes dele ser morto.

Loló: Vi a Lulu sozinha longe do corpo, e por isso ela não é a impostora.

Lulu: Eu e Lalá ficamos juntos o tempo inteiro. 
Lilão: Vi Lili voltando da direção contrária do corpo antes de encontrá-lo e reportar.

Após a votação, os participantes eliminaram Lili para fora da nave. Considerando que nessa partida, só havia um impostor, e esse não era Lelé e apenas o impostor mentiu, os tripulantes conseguiram eliminar o impostor?

\subsubsection{Discussão da solução de estudante premiado na OBMEP}

Diante da atividade acima, o estudante informou a seguinte resposta: "Não, porque Loló disse que viu Lulu sozinha, porém Lulu e Lalá disseram que estavam juntos o tempo todo, levando ao fato de que Loló estaria mentindo".

Com a frase retratada desta maneira, é possível observar que o estudante apresentou um processo de decomposição das respostas apresentadas, reconhecimento de padrões entre respostas que falam da mesma pessoa e abstração para observar qual destas respostas entra em divergência com as demais.

A presença dos pilares do pensamento computacional ficam evidentes na atividade: decomposição, ao entender as afirmações de cada um; abstração, analisando as informações individuais; para assim reconhecer um padrão e executar um algoritmo até encontrar a informação que não segue o padrão apresentado. Vale ressaltar que a ordem que os pilares se apresentam em uma atividade pode variar de acordo com a mesma, bem como seu grau de complexidade ou passos necessários. A importância do processo de aprendizagem é o fator determinante para o rendimento do estudante, e nesse sentido os pilares auxiliam na compreensão dos educandos e na avaliação dos educadores.

Diante desta atividade, foram discutidas na turma do programa Mentores da OBMEP as diferentes formas que poderiam ser utilizadas para a resolução de um mesmo problema, podendo ser inseridas tabelas verdade da lógica clássica, ou a teoria dos grafos. Entretanto, mesmo com estudantes do ensino médio, que não tiveram contato com nenhum desses conceitos na escola básica, é possível observar uma resolução concreta, justamente devido a implementação do pensamento computacional que induz o educando a resolver utilizando os seus quatro pilares.

\section{Conclusão}

O presente artigo é um recorte de uma pesquisa que busca entender o diferencial das questões de matemática que abordam o pensamento computacional. Vale ressaltar que este, por sua vez, está previsto para ser trabalhado na escola básica, de forma obrigatória no ensino fundamental e facultativa no ensino médio. Entretanto, ele é um conceito presente em questões de qualquer conteúdo, e não apenas um conteúdo isolado. As estruturas cognitivas construídas no estudante ao interpretar uma questão e resolvê-la com os pilares do pensamento computacional tendem promover uma melhor compreensão não apenas dos conteúdos curriculares, mas também o funcionamento de instrumentos de seu cotidiano, como um smartphone.

Analisando as resoluções enviadas pelos estudantes, fica clara a amplitude da matemática na diversidade de resolução de problemas, e que estes, por sua vez, estão 
intimamente ligados à construção de algoritmos quanto à generalização e a análise de $\mathrm{n}$ casos de um enunciado.

Todavia, se faz necessária a compreensão docente para que isso seja possível. Cada escola possui uma realidade e cada estudante uma necessidade, compreendida pelo docente mediador do conhecimento. Por esta razão, os argumentos discutidos até então, bem como as 19 atividades realizadas, serão divulgadas aos docentes da região de execução da pesquisa, bem como todos que tiverem interesse em inovar os métodos educacionais em suas aulas de matemática.

\section{Referências}

Barr, V. Stephenson, C. (2011) Bringing computational thinking to K-12: what is Involved and what is the role of the computer science education community?. Communications of the ACM, v. 2, n.1, págs 44-54. Disponível em: https://dl.acm.org/doi/10.1145/1929887.1929905. Acesso em: 05 jul. 21.

BNCC. (2018). Base Nacional Comum Curricular. Disponível em: http://basenacionalcomum.mec.gov.br/images/BNCC_EI_EF_110518_versaofinal_si te.pdf. Acesso em: 08 mar. 21.

Bobsin, R. S.; Nunes, N. B.; Kologeski, A. L.; Bona, A. S. D. O Pensamento Computacional presente na Resolução de Problemas Investigativos de Matemática na Escola Básica. In: Simpósio Brasileiro de Informática na Educação, 31. , 2020, Online. Anais [...]. Porto Alegre: Sociedade Brasileira de Computação, 2020 . p. 1473-1482. DOI: https://doi.org/10.5753/cbie.sbie.2020.1473.

Bona, A. S. (2016) Aulas Investigativas e a construção de conceitos de matemática: um estudo a partir da teoria de Piaget. Curitiba: CRV.

Bona, A. S. D, Bobsin, R. S, Kologeski, A. L. (2020) "Contextualizando a matemática em oficinas com o pensamento computacional". Revista Educação em Foco: Tecnologias digitais \& inovação em práticas de ensino. n. 1 vol. 1. págs. 189-206. e-Publicar: Rio de Janeiro, Brasil.

CGEE. (2011). Avaliação do impacto da Olimpíada Brasileira de Matemática nas escolas públicas. Centro de Gestão e Estudos Estratégicos. v. 11. Brasília, Brasil. Disponível em: http://server22.obmep.org.br:8080/media/servicos/recursos/251395.o. Acesso em: 05 mar. 21.

Duarte, A.; Moreira, H.; Mello, T. S. Competitividade como Fator Motivacional para o Estudo de Computação. Brazilian Symposium on Computers in Education (Simpósio Brasileiro de Informática na Educação - SBIE), [S.1.], out. 2012. ISSN 2316-6533. Disponível em: <https://br-ie.org/pub/index.php/sbie/article/view/1525/1290>. Acesso em: 26 jul. 2021. doi:http://dx.doi.org/10.5753/cbie.sbie.2010.\%p. 
Fomin, D., Genkin, S., Itenberg, I. (2012), Círculos Matemáticos - A experiência russa. Instituto de Matemática Pura e Aplicada: Rio de Janeiro, Brasil.

Guarda, G. F.; Pinto, S. C. C. S.. Dimensões do Pensamento Computacional: conceitos, práticas e novas perspectivas. In: Simpósio Brasileiro de Informática na Educação, 31. , 2020, Online. Anais [...]. Porto Alegre: Sociedade Brasileira de Computação, 2020 . p. 1463-1472. DOI: https://doi.org/10.5753/cbie.sbie.2020.1463.

Nunes, N. B.; Bona, A. S. D. O pensamento computacional e algumas questões da OBMEP como Metodologias inovadoras para as aulas de Matemática. In: Encontro Nacional de Computação dos Institutos Federais (EnCompIF), 8. , 2021, Evento Online. Anais do XLI Congresso da Sociedade Brasileira de Computação. Porto Alegre: Sociedade Brasileira de Computação, 2021 . p. 9-12. ISSN 2763-8766. DOI: https://doi.org/10.5753/encompif.2021.15943.

OBMEP. (2021). Disponível em: http://www.obmep.org.br/apresentacao.htm. Acesso em: 05 jul. 21.

Palfrey, J. e Gasser, U. (2011). Nascidos na era digital: entendendo a primeira geração de nativos digitais. Artmed: Porto Alegre, Brasil.

Ponte, J. P. Brocardo, J. Oliveira, H. (2006). Investigações matemáticas na sala de aula. Autêntica.

SAEB. (2017). Saeb 2017 revela que apenas 1,6\% dos estudantes brasileiros do Ensino Médio demonstraram níveis de aprendizagem considerados adequados em Língua Portuguesa. Disponivel

em: https://www.gov.br/inep/pt-br/assuntos/noticias/saeb/saeb-2017-revela-que-apenas-1 6-dos-estudantes-brasileiros-do-ensino-medio-demonstraram-niveis-de-aprendizage m-considerados-adequados-em-lingua-portuguesa. Acesso em: 05 jul. 21.

Santana, T.; Lopes, A.; Braga, A. Uma análise de correlação entre disciplinas do ensino fundamental e o desempenho na Olimpíada Brasileira de Informática. Brazilian Symposium on Computers in Education (Simpósio Brasileiro de Informática na Educação - SBIE), [S.1.], p. 557, nov. 2019. ISSN 2316-6533. Disponível em: $<$ https://br-ie.org/pub/index.php/sbie/article/view/8760/6321>. Acesso em: 26 jul. 2021. doi:http://dx.doi.org/10.5753/cbie.sbie.2019.557.

Silva, L. C. L. (2019) A relação do Pensamento Computacional com o ensino de Matemática na Educação Básica.Dissertação (mestrado) -Universidade Estadual Paulista (Unesp), Faculdade de Ciências e Tecnologia, Presidente Prudente. Disponível em: https://repositorio.unesp.br/bitstream/handle/11449/191251/silva_lcl_me_sjrp.pdf?se quence $=5 \&$ isAllowed $=\mathrm{y}$. Acesso em: 05 jul.21

Souza, J. S.; Lopes, A. S. B. Estimulando o pensamento computacional e o raciocínio lógico no ensino fundamental por meio da OBI e computação desplugada. Brazilian Symposium on Computers in Education (Simpósio Brasileiro de Informática na Educação - SBIE), [S.1.], p. 1893, out. 2018. ISSN 2316-6533. Disponível em: 
$<$ https://br-ie.org/pub/index.php/sbie/article/view/8186/5865>. Acesso em: 26 jul. 2021. doi:http://dx.doi.org/10.5753/cbie.sbie.2018.1893.

Vicari, R. et. al., Pensamento Computacional - Revisão Bibliográfica. Universidade Federal do Rio Grande do Sul, Porto Alegre/RS, 2020.

Wing, J. M. (2006) Computational thinking. Communications of the ACM, v. 49, n. 3, págs. 33-35. Disponível em: dl.acm.org/citation.cfm?id=1118215. Acesso em: 05 mar. 21.

Wing, J. M. (2010) Computational thinking:what and why? Disponível em: http://www.cs.cmu.edu/ CompThink/resources/TheLinkWing.pdf. Acesso em: 19 jun. 20. 